\title{
SEX DIFFERENCES IN SHOCK ATTENUATION DURING RUNNING
}

\author{
Jonathan Sinclair \\ Centre for Applied Sport \& Exercise Sciences, School of Sport \& Wellbeing, College of Health \& Wellbeing, \\ University of Central Lancashire, United Kingdom \\ Address for correspondence: \\ Jonathan Sinclair \\ Darwin Building \\ Centre for Applied Sport Exercise and Nutritional Sciences \\ School of Sport \& Wellbeing, College of Health \& Wellbeing \\ University of Central Lancashire \\ Preston, Lancashire PR1 2HE, United Kingdom \\ E-mail: jksinclair@uclan.ac.uk
}

\begin{abstract}
Ahstract The current investigation was conducted to determine whether sex differences in skeletal accelerations and shock attenuation were evident during running. Twelve male and twelve female recreational runners ran at $4.0 \mathrm{~m} . \mathrm{s}^{-1}$. Axial accelerations were measured at $1,000 \mathrm{~Hz}$ using accelerometers mounted at the tibia and sacrum. Peak tibial and sacrum axial accelerations were obtained and utilized to calculate the extent of shock attenuation. The results showed that peak sacrum accelerations were significantly larger in female runners $(5.16 \pm 0.64 \mathrm{~g})$ compared to males $(4.37 \pm 0.75 \mathrm{~g})$. It was also shown that shock attenuation $(31.90 \pm 19.85 \%)$ was significantly lower in female runners in relation to males $(47.89 \pm 11.46 \%)$. The findings from the current investigation indicate that female runners experience greater skeletal accelerations which may place greater stress on the musculoskeletal structures required attenuate transients forces which can be detrimental to passive tissues.
\end{abstract}

Key worlds running, gender, shock attenuation

\section{Introduction}

Both recreational and competitive distance runners are renowned for their susceptibly to chronic injuries (Taunton et al., 2002), with as many as $80 \%$ experiencing an injury each year (van Gent et al., 2007). The aetiology of chronic running injuries is extremely complex and the factors linked to the development of injuries are often poorly understood. However, it is generally considered that running related pathologies relate to repetitive loading of the lower extremities (Robbins, Hanna, 1987; Whittle, 1999). Female runners have been identified as being at increased risk from chronic running injuries in relation to age matched males (Taunton et al., 2002).

It is postulated that sex differences in chronic injury susceptibility relates to the distinct kinetic and kinematic parameters exhibited by female runners (Sinclair, Greenhalgh, Edmundson, Brooks, Hobbs, 2012). Although females are regarded as being at increased risk from running injuries the specific aetiological contributors are not well understood. Thus, there are requirements for further examination into the biomechanical mechanisms that may 
be associated with the aetiology of injury in female runners. Only a small number of investigations to date have investigated biomechanical differences between sexes during running.

Malinzak, Colby, Kirkendall, Yu, Garrett (2001) investigated gender differences in coronal and sagittal plane knee motion. It was demonstrated that the whilst the coronal plane knee excursion was similar between genders, women were found to exhibit less peak knee flexion and a lower range of motion in the knee compared to men. Ferber, Davis, Williams (2003) examined the gender differences in 3-D kinematics of the hip and knee. Female runners exhibited greater peak hip adduction, hip internal rotation and knee abduction compared to men. Sinclair et al. (2012) examined the kinetics and 3-D kinematics of running, their findings showed that whilst no differences in kinetics were evident, female runners exhibited an increased knee abduction and internal rotation. Sinclair, Selfe (2015) investigated sex differences in patellofemoral loading during the stance phase of running. They found that females were associated with significantly greater patellofemoral loads than male runners.

Each foot contact during running causes a transient impulse propagates through the musculoskeletal system (Whittle, 1999). The impact transient generated as a function of footstrike is attenuated by the musculoskeletal structures in order to abate accelerations at the skull, which would otherwise lead to disorders of the vestibular and visual systems (Hamill, Derrick, Holt, 1995). Shock attenuation is therefore an essential parameter when quantifying impact transients during running, as it serves a quantifiable indicator of the extent to which the impact shock wave is reduced (Shorten, Winslow, 1992). The mechanical process by which shock is attenuated is through the absorption of energy via muscle contraction, lower extremity alignment and deformation of passive musculoskeletal structures (Mercer et al., 2010). It has been shown that can be mediated by a number of different biomechanical/ anthropometrical parameters (Mercer et al., 2010). However there is current no research which has examined sex differences in shock attenuation during running.

Therefore, the aim of the current investigation was to examine sex differences in skeletal accelerations and shock attenuation during running. A study of this nature may provide further insight into the distinct chronic injury profiles of male and female runners.

\section{Methods}

\section{Participants}

Twelve male and twelve female runners volunteered for this investigation. The mean characteristics of the participants were males; age age $25.1 \pm 4.0$ years, height $1.77 \pm 0.1 \mathrm{~m}$ and body mass $73.2 \pm 6.5 \mathrm{~kg}$ and females; age $24.2 \pm 4.5$ years, height $1.66 \pm 0.1 \mathrm{~m}$ and body mass $64.3 \pm 6.4 \mathrm{~kg}$. Runners were free from musculoskeletal pathology at the time of data collection and gave written informed consent. The procedure utilized for this investigation was approved by a University ethical committee.

\section{Procedure}

Participants ran at a velocity of 4.0 m.s $^{-1} \pm 5 \%$, striking an embedded piezoelectric force platform (Kistler, Kistler Instruments Ltd., Alton, Hampshire) with their right (dominant) foot (Sinclair, Hobbs, Taylor, Currigan, Greenhalgh, 2014). Running velocity was quantified using infrared timing gates (Newtest, Oy Koulukatu, Finland). The stance phase was delineated as the duration over which $>20 \mathrm{~N}$ of vertical force was applied to the force platform (Sinclair, Edmundson, Brooks, Hobbs, 2011). Runners were required to complete five successful trials. 
To quantify accelerations at the tibia and sacrum, two accelerometers (Biometrics ACL 300, Gwent United Kingdom) sampling at $1,000 \mathrm{~Hz}$ were utilized. The accelerometers were both positioned onto a piece of lightweight carbon-fibre which is the same as the mounting protocol documented by Sinclair, Greenhalgh, Edmundson, Brooks, Hobbs (2013). The tibial accelerometer was attached securely to the anterio-medial aspect of the distal tibia in line with its longitudinal axis $8 \mathrm{~cm}$ above the malleolus (Sinclair, Bottoms, Taylor, Greenhalgh, 2010). The sacrum accelerometer was positioned onto the bony prominence of the sacrum and secured using an elastic belt (Mizrahi, Verbitsky, Isakov, Daily, 2000). The sacrum accelerometer was positioned so that its vertical axis aligned with the longitudinal axis of the spine. Ground reaction force and acceleration data were collected synchronously using an analogue to digital interface board.

\section{Processing}

The acceleration signals were filtered with a $60 \mathrm{~Hz}$ low-pass Butterworth 4th order zero-lag filter. Peak tibial acceleration was defined as the highest positive acceleration peak measured during the first $20 \%$ of the stance phase. Peak sacrum acceleration delineated as the highest positive acceleration peak during the first $50 \%$ of stance. Acceleration slope at each location was quantified by dividing acceleration peak by the duration over which the acceleration occurred. With the data obtained above shock attenuation was also calculated using the formula outlined below.

Shock attenuation \% = [1 - (peak sacrum acceleration / peak tibial acceleration $) \times 100]$.

\section{Analysis}

Statistical differences in acceleration and attenuation parameters between sexes were explored using independent samples t-tests, with significance accepted at the $P \leq 0.05$ level (Sinclair et al., 2013). All statistical tests were conducted using SPSS v22.0 (SPSS Inc., Chicago, USA).

\section{Results}

Table 1 and Figure 1 present sex differences in skeletal acceleration and shock attenuation parameters. The results show statistical differences between sexes for acceleration and attenuation parameters.

Table 1. Sex differences in skeletal accelerations and shock attenuation

\begin{tabular}{lrrrr}
\cline { 2 - 5 } & \multicolumn{2}{c}{ Male } & \multicolumn{2}{c}{ Female } \\
\cline { 2 - 5 } & Mean & SD & Mean & \multicolumn{1}{c}{ SD } \\
\hline Peak tibial acceleration (g) & 8.94 & 2.33 & 8.64 & 3.49 \\
Peak sacrum acceleration (g) & 4.37 & 0.75 & 5.16 & 0.64 \\
Time to peak tibial acceleration (s) & 0.05 & 0.04 & 0.05 & 0.03 \\
Time to peak sacrum acceleration (s) & 0.07 & 0.01 & 0.08 & 0.01 \\
Tibial acceleration slope (g/s) & 309.24 & 139.98 & 324.37 & 182.33 \\
Sacrum acceleration slope (g/s) & 67.91 & 9.62 & 64.79 & 6.59 \\
Shock attenuation (\%) & 47.89 & 11.46 & 31.90 & 19.85 \\
\hline
\end{tabular}




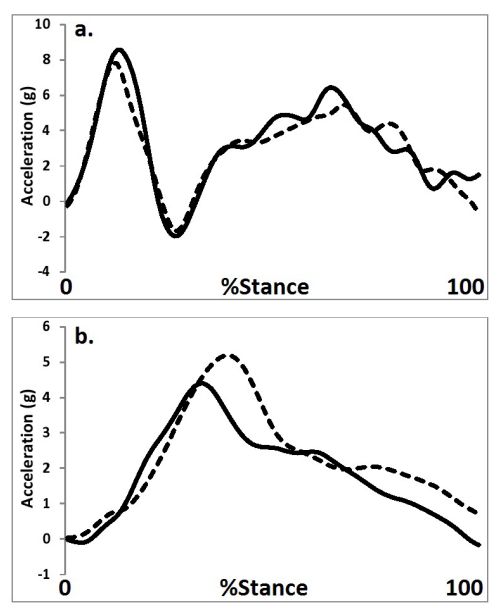

Figule 1. Tibial (a) and sacrum (b) acceleration parameters as a function of sex (black = male \& female = dash)

A main effect for peak sacrum acceleration was observed $\left(t_{(22)}=2.77, P<0.05\right)$, with females being associated with significantly greater accelerations than males (Table 1; Figure 1b). A main effect was also shown for shock attenuation $\left(\mathrm{t}_{(22)}=2.42, \mathrm{P}<0.05\right)$, with males exhibiting significantly greater shock attenuation compared to females (Table 1). No further significant differences were evident.

\section{Discussion}

The aim of the current investigation was to examine sex differences in shock attenuation during running. To the authors knowledge this represents the first comparative investigation of skeletal accelerations and attenuation between sexes. Research of this nature may provide further information regarding aetiological factors that contribute to the distinct chronic injury patterns in males and female runners.

The first key finding from the current investigation is that sacrum accelerations were significantly larger in female runners in comparison to males. This finding may have important implications as clinical research has linked the magnitude of skeletal impact transients to the initiation of lower back pathologies in runners (Voloshin, Wosk, 1992). Aetiological literature has also shown that female runners are more susceptible to stress fractures than age matched males (Miletic et al., 2012). Specifically it has been demonstrated that sacral stress fractures occur more frequently in female runners (Pester, Smith, 1992). The current investigation therefore supports the conjecture that female runners are at increased risk from stress fractures and low back pain and may provide new information regarding the mechanical stimuli that contribute to these pathologies.

Of further importance is the observation that shock attenuation was significantly lower in female runners in comparison to males. Therefore, it can be speculated that greater shock transmission from tibia to sacrum may lead to greater propensity for chronic damage as passive musculoskeletal structures endure increased stress. The findings from this study oppose those from Dufek, Mercer, Griffin (2009) who showed that female runners exhibited greater shock attenuation. It is proposed that this divergence between studies may be due to the reduced 
velocities that the participants of Dufek et al., were tested under in relation to the current study. The increased running speed from the current study may have placed increased stress on the musculoskeletal system. Therefore a distinct strategy of attenuating impact accelerations may be evident during higher running speeds (Dufek et al., 2009). Shock attenuation represents the mechanical process by which transient energy diminishes as it propagates through the musculoskeletal system (Valiant, 1990). Shock attenuation is achieved via muscle contraction, lower body alignment and passive musculoskeletal tissue deformation (Mercer et al., 2010). A further discrepancy is that Dufek et al. (2009) quantified transient attenuation from the tibia to the head as opposed to the sacrum. This indicates that females may attenuate transient accelerations to a greater extent from tibia and head but to a reduced degree between tibia to sacrum. This observation may therefore provide further insight into the increased susceptibility of females to lower extremity chronic pathologies (Taunton et al., 2002), although further prospective analyses are required with shock attenuation as a designated risk factor before this can be substantiated clinically.

A potential limitation of the current study is that the potential effects of wobbling mass on skeletal accelerations were not accounted for. Challis, Pain (2008) proposed that tissue artefact can affect skeletal accelerations during dynamic activities. It can thus be speculated that females runners examined as part of the current investigation may have had a higher percentage of adipose tissue, and as such comparative results of shock attenuation between sexes may have be influenced by this (Dufek et al., 2009).

In conclusion, whilst sex differences in running biomechanics have been investigated previously there has yet to be any published work which has examined shock attenuation in between sexes. The current study addresses this by providing a comparative investigation of skeletal accelerations and shock attenuation in male and female runners. The current investigation showed that sacrum accelerations were greater and shock attenuation was significantly lower in female runners. The findings from the current investigation indicate that female runners experience greater skeletal accelerations which may place greater stress on the musculoskeletal structures required attenuate transients forces which can be detrimental to passive tissues.

\section{References}

Challis, J.H. Pain, M.T.G. (2008). Soft tissue motion influences skeletal loads during impacts. Exercise and Sports Science Reviews, $36,71-75$.

Dufek, J.S., Mercer, J.A., Griffin, J.R. (2009). The effects of speed and surface compliance on shock attenuation characteristics for male and female runners. Journal of Applied Biomechanics, 25, 219-228.

Ferber, R., Davis, I.M., Williams, D.S. (2003). Gender differences in lower extremity mechanics during running. Clinical Biomechanics, $18,350-357$.

Hamill, J., Derrick, T.R., Holt, K.G. (1995) Shock attenuation and stride frequency during running. Human Movement Science, 14, $45-60$.

Malinzak, R.A., Colby, S.M., Kirkendall, D.T., Yu, B., Garrett, W.E. (2001). A comparison of knee joint motion patterns between men and women in selected athletic tasks. Clinical Biomechanics, 16, 438-445.

Mercer, J.A., Dufek, J.S., Mangus, B.C., Rubley, M.D., Bhanot, K., Aldridge, J.M. (2010). A description of shock attenuation for children running. Journal of Athletic Training, 45, 259-264.

Miletic, D., Sestan, B., Pusic, M., Cicvarić, T., Tudor, A., Roth, S., Santic, V. (2012). Unusual consecutive sacral stress fractures in a female distant runner: a case report. European journal of physical and rehabilitation medicine, 48, 283-287.

Mizrahi, J., Verbitsky, O., Isakov, E., Daily, D. (2000). Effect of fatigue on leg kinematics and impact acceleration in long distance running. Human Movement Science, 19, 139-151.

Pester, S., Smith, P. C. (1992). Stress fractures in the lower extremities of soldiers in basic training. Orthopaedic review, 21, 297-303. 
Robbins, S.E., Hanna, A.M. (1987). Running-related injury prevention through barefoot adaptations. Medicine \& Science in Sports \& Exercise, 19, 148-156.

Shorten, M.R., Winslow, D.S. (1992). Spectral analysis of impact shock during running. International Journal of Sports Biomechanics, 8, 288-304.

Sinclair, J., Greenhalgh, A., Edmundson, C.J., Brooks, D., Hobbs, S.J. (2012). Gender differences in the kinetics and kinematics of distance running: implications for footwear design. International Journal of Sports Science \& Engineering, 6, 118-128.

Sinclair, J., Hobbs, S.J., Taylor, P.J., Currigan, G., Greenhalgh, A. (2014). The influence of different force measuring transducers on lower extremity kinematics. Journal of Applied Biomechanics, 30, 166-172.

Sinclair, J., Edmundson, C.J., Brooks, D., Hobbs, S.J. (2011). Evaluation of kinematic methods of identifying gait Events during running. International Journal of Sport Science \& Engineering, 5, 188-192.

Sinclair, J, Greenhalgh, A., Edmundson, C.J., Brooks, D., Hobbs, S.J. (2013). The influence of barefoot and barefoot-inspired footwear on the kinetics and kinematics of running in comparison to conventional running shoes. Footwear Science, 5, 45-53.

Sinclair, J., Bottoms, L., Taylor, K., Greenhalgh, A. (2010). Tibial shock measured during the fencing lunge: the influence of footwear. Sports Biomechanics, 9, 65-71.

Sinclair, J., Taylor, P.J., Hobbs, S.J. (2013). Alpha level adjustments for multiple dependent variable analyses and their applicability a review. International Journal of Sport Science \& Engineering, 7, 17-20.

Sinclair, J., Selfe, J. (2015). Sex differences in knee loading in recreational runners. Journal of biomechanics, 48 (10), $2171-2175$.

Taunton, J.E., Ryan, M.B., Clement, D.B., McKenzie, D.C., Lloyd-Smith, D.R., Zumbo, B.D. (2002). A retrospective case-control analysis of 2002 running injuries. British Journal of Sports Medicine, 36, 95-101.

van Gent, R.N., Siem, D., van Middelkoop, M., van Os, A.G., Bierma-Zeinstra, S.M.A., Koes, B.W. (2007). Incidence and determinants of lower extremity running injuries in long distance runners: a systematic review. British Journal of Sports Medicine, 41, 469-480.

Voloshin, A., Wosk, J. (1982). An in vivo study of low back pain and shock absorption in the human locomotor system. Journal of Biomechanics, 15, 21-27.

Whittle, M.W. (1999). The generation and attenuation of transient forces beneath the foot; a review. Gait \& Posture, 10, $264-275$.

Cite this article aS: Sinclair, J. (2016). Sex Differences in Shock Attenuation during Running. Central European Journal of Sport Sciences and Medicine, 15 (3), 37-42. DOI: 10.18276/cej.2016.3-04. 